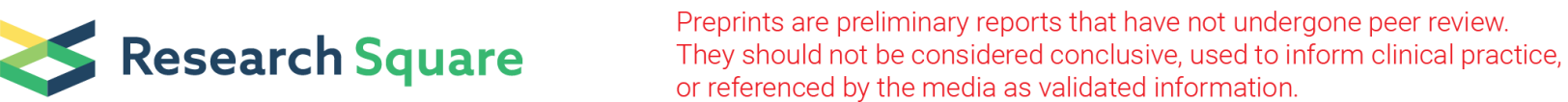

\section{Construction of a high-density genetic map and identification of quantitative trait loci for nitrite tolerance in the Pacific white shrimp (Litopenaeus vannamei)}

\section{Digang Zeng}

guangxi academy of Fisheries Sciences

Xiuli Chen

Guangxi Academy of Fisheries Sciences

\section{Weilin Zhu}

Guangxi Academy of Fisheries Sciences

\section{Min Peng}

Guangxi Academy of Fisheries Sciences

\section{Chunling Yang}

Guangxi Academy of Fisheries Sciences

Qingyun Liu

Guangxi Academy of Fisheries Sciences

\section{Qiangyong Li}

Guangxi Academy of Fisheries Sciences

\section{Huanling Wang}

Huazhong Agriculture University

Hong Liu

Huazhong Agriculture University

Jingzhen Liang

Guangxi University of Science and Technology

\section{Yong Lin}

Guangxi Academy of Fisheries Sciences

Xiaohan Chen ( $\sim$ gxnnscs@126.com )

Guangxi Academy of Fisheries Sciences

Yongzhen Zhao ( $\square$ yongzhenzhao@hotmail.com )

Guangxi Academy of Fisheries Sciences https://orcid.org/0000-0002-9051-0383

\section{Research article}

Keywords: genetic map, QTL, transcriptomic, nitrite tolerance, Litopenaeus vannamei 
Posted Date: December 16th, 2019

DOI: https://doi.org/10.21203/rs.2.18940/v1

License: (1) This work is licensed under a Creative Commons Attribution 4.0 International License. Read Full License 


\section{Abstract}

\section{Background}

Nitrite is a major environmental toxin in aquaculture systems disrupting multiple physiological functions in aquatic animals. Although nitrite tolerance in shrimp is closely related to successful industrial production, few genetic studies of this trait are available.

\section{Results}

In this study, we constructed a high-density genetic map of $L$. vannamei with 17,242 single nucleotide polymorphism markers spanning $6,828.06$ centimorgans ( $\mathrm{CM}$ ) and an average distance of $0.4 \mathrm{cM}$ between adjacent markers on 44 linkage groups (LGs). Using this genetic map, we identified 2 quantitative trait loci (QTLs) involved in nitrite tolerance on two LGs explaining $8.42-10.31 \%$ of the phenotypic variance. We then sequenced the transcriptomes of the most nitrite-tolerant and the most nitrite-sensitive individuals from each of four genetically distinct $L$. vannamei families and found 2,002, 1,983,1,954, and 1,867 differentially expressed genes in the four families, respectively. By integrating QTL and transcriptomics analyses, we identified a candidate gene associated with nitrite tolerance. This gene was annotated as solute carrier family 26 member 6 (SLC26A6). RNA interference (RNAi) analysis demonstrated that SLC26A6 was critical for nitrite tolerance in L. vannamei.

\section{Conclusions}

The present study increases our understanding of the molecular mechanisms underlying nitrite tolerance in shrimp, and provides a basis for molecular-marker-assisted shrimp breeding.

\section{Background}

The Pacific white shrimp (Litopenaeus vannamei) is the world's most extensively cultivated shrimp species [1, 2]; annual production of $L$. vannamei accounts for about $50 \%$ of all penaeid shrimp production [3]. In 2016, the global production of L. vannamei approached 4.16 million tons [3]. However, the intensive or semi-intensive farming model commonly used at present for shrimp aquaculture often leads to water quality deterioration, and the resulting toxicological stress causes mortality or disease in farmed shrimp, negatively affecting breeding efficiency [4].

Nitrite, a strong oxidant that is the intermediate product of the biological oxidation of ammonia to nitrate [5], is one of the most common toxins in aquaculture water [6]. Exposure to nitrite decreases oxyhemoglobin levels in mammals, leading tissue hypoxemia and hypoxia $[7,8]$. As the prosthetic group of the hemocyanin in crustacean blood is also a copper-containing compound, hemocyanin may behave similarly to hemoglobin; therefore, nitrite exposure in crustaceans might lead to hypoxia, cyanosis, or even death $[9,10]$. Indeed, it has been reported that nitrite has serious deleterious effects on shrimp growth [11], immunity [12] and survival $[11,13,14]$. Therefore, the maintenance of low aquatic nitrite concentrations in required for successful shrimp farming. However, aquaculture water quality is affected by many factors, 
including weather and artificial feeding [15], and efforts to control nitrite levels in aquaculture water are not always effective. Although the establishment of new, nitrite-tolerant shrimp varieties might represent an effective solution to this problem, such breeding programs require a prior understanding of the genetic basis of nitrite tolerance in shrimp. Unfortunately, the genetic basis of nitrite tolerance in shrimp has yet to be characterized.

Genetic maps play an important role in the identification of QTLs and functional genes associated with economically-valuable traits in plants and animals [16]. In addition, genetic maps can be used to assemble sequences for genome sequencing, genome structure comparison, and marker-assisted selection (MAS) [17-19]. Previously, genetic map construction was primarily based on traditional molecular marker techniques, such as random amplified polymorphic DNA (RAPD), restriction fragment length polymorphism (RFLP), amplified fragment length polymorphism (AFLP), simple sequence repeat (SSR), and (inter-simple sequence repeat) ISSR [20-22]. However, the density of the genetic maps generated based on these traditional markers was relatively low, and most of these genetic maps contained only a few hundred markers. The fusion of second-generation sequencing technologies with genotyping methods, including genotyping-by-sequencing (GBS) [23], restriction site-associated DNA sequencing (RAD-seq) [24], and specific length amplified fragment sequencing (SLAF-seq), allow the identification of millions of SNPs in plant and animal genomes. In addition, these technologies produce markers that are denser, more consistent, more efficient, and less costly than traditional methods [25].

To understand the genetic mechanisms underlying nitrite tolerance in shrimp, and to identify related candidate genes, we constructed a high-density single nucleotide polymorphism (SNP) genetic map of $L$. vannamei using SLAF-seq. Using this genetic map in conjunction with phenotypic data (survival time under acute nitrite stress), we identified quantitative trait loci (QTLs) for nitrite tolerance in $L$. vannamei. We further compared transcriptomic differences between nitrite-tolerant and nitrite-sensitive $L$. vannamei from genetically-distinct families using RNA sequencing (RNA-seq). We then performed a comprehensive analysis of gene expression profiles and QTLs to identify candidate genes associated with nitrite tolerance. Finally, we verified candidate genes using RNA interference (RNAi).

\section{Methods}

\section{Establishment of the mapping family}

The $L$. vannamei family used for mapping was established at the National and Guangxi Shrimp Genetic Breeding Center (Guangxi Province, China). To develop the mapping family, a male shrimp from a family with a relatively strong nitrite tolerance and a female shrimp from a normal family were artificially inseminated, and their progeny were cultured in a pond. After cultivation for one year, a male shrimp and a female shrimp were randomly selected from the progeny and artificially inseminated. Their progeny (the F1 population) were used as the mapping family (LV-1).

\section{Acute nitrite stress test}


We randomly selected 157 shrimp from the mapping family (eight-months-old; average mass: $20.46 \mathrm{~g}$ ). Selected shrimp were cultured in a $2 \mathrm{~m} \times 4 \mathrm{~m} \times 1 \mathrm{~m}$ indoor pool. The pool water remained aerated throughout the acclimation period and the experiment, with $\mathrm{pH}$ maintained at $8.2 \pm 0.3$; temperature maintained at $27.0 \pm 0.5^{\circ} \mathrm{C}$; salinity maintained at $30.1 \%$; and dissolved oxygen maintained at 7-8 mg/L. All shrimp were fed formulated pellets (Zhengda Corporation, China) daily at a ratio of $5 \%$ of average body weight. Shrimp were allowed to acclimate for $5 \mathrm{~d}$ before the acute nitrite stress test. Analytically-pure $\mathrm{NaNO}_{2}$ was dissolved in filtered seawater to prepare a concentrated nitrite stock solution; this stock solution was then added to the pool water to increase the nitrite concentration to $700 \mathrm{mg} / \mathrm{L}$. This concentration was chosen because preliminary experiments (Additional file 1: Table S1) indicated that all experimental shrimp will die within 80 hours at nitrite concentration of $700 \mathrm{mg} / \mathrm{L}$, and this time range was suitable for observation. The nitrite concentration in the pool was measured every $24 \mathrm{~h}$ using the standard method [26]. Filtered seawater or nitrite stock solution was added to the pool as needed to maintain the nitrite concentration at $700 \mathrm{mg} / \mathrm{L}$. Dead shrimp were collected every $1 \mathrm{~h}$. Shrimp were considered dead when lying on the bottom of the pool out of balance and unreactive when touched with a wooden stick. Survival time was recorded to represent nitrite tolerance. Dead shrimp were collected immediately upon observation, frozen in liquid nitrogen, and transferred to an ultra-low temperature freezer $\left(-80^{\circ} \mathrm{C}\right)$ until DNA extraction.

\section{DNA extraction, SLAF library preparation, and sequencing}

We extracted DNA from the tail muscles of the 157 selected F1 progeny and the two parents using Marine Animal DNA Extraction kits (Tiangen, China). The quality and quantity of each extracted DNA sample was measured using 1\% agarose gel electrophoresis and a ND2000 spectrophotometer (NanoDrop, USA), respectively. We used restriction enzyme digestion prediction software (Biomarker Technologies Corporation, China) to predict appropriate endonucleases for genomic fragmentation based on the genome of L. vannamei (https://www.ncbi.nlm.nih.gov/genome/?term=Vannamei) [27]. Based on this prediction, all genomic DNA samples were digested with the enzymes Haell and Hpy166II. The dual-index adapter was then ligated to the fragments using T4 ligase, and polymerase chain reactions (PCRs) were performed. Amplification products (314-414 bp, including the adapter) were purified and collected using gel extraction kits (Illumina, USA). These fragments were the re-amplified for SLAF sequencing using PCR. SLAF sequencing was performed using an Illumina HiSeq system (Illumina, USA), following the manufacturer's instructions. To evaluate the accuracy of library construction, we used the same procedures to construct SLAF sequence libraries for Oryza sativa L. japonica as a control. All library construction and sequencing were performed at Biomarker Technologies Corporation (Beijing, China).

\section{SLAF-seq data analysis and genotyping}

First, the raw sequencing data were assigned to samples based on the dual-index adapters. These adapter sequences were removed, and the reads were filtered to remove all reads containing the adapter sequence, as well as reads containing more than 10\% unknown $(\mathrm{N})$ bases. As the first few bp of each read were the residue left by the enzyme fragment, the sequencing quality in this area was low. Therefore, only bases 4$103 \mathrm{bp}$ were analyzed (fragment length: $100 \mathrm{bp}$ ). The filtered clean reads were compared to the $L$. 
vannamei genome (https://www.ncbi.nlm.nih.gov/genome/?term=Vannamei) [27] using BWA software[28]. Reads with $>95 \%$ identity, and where both ends matched to the same location in the $L$. vannamei genome, were considered to be derived from the same SLAF marker. SNP-based polymorphic SLAF markers were identified by comparing reads derived from the same SLAF marker. These polymorphic SLAF markers were filtered to remove markers whose parental sequencing depth was less than 10x; markers with $>5$ SNPs; markers for which the proportion of genotypes covering all offspring was $<70 \%$; and markers with severe partial segregation (chi-squared test $\mathrm{P}$-value $<0.05$ ). The remaining polymorphic SLAF markers were encoded into eight segregation patterns: ab × cd; ef × eg; hk × hk; Im × Il; nn × np; aa $\times$ bb; ab × cc; and cc $\times$ ab. As the mapping population we used was an F1 population, the polymorphic SLAF markers with the aa $x$ bb separation pattern were discarded, and the remaining polymorphic SLAF markers were used to construct the linkage map.

\section{Genetic map construction and QTL analysis}

The genetic map was constructed with iterative ordering and error correction, as implemented in HighMap [29]. The polymorphic SLAF markers were imported into HighMap, and the linkage groups were determined using the pair-wise logarithm of the odds (LOD) test. The order of the markers in the linkage group was calculated using the enhanced Gibbs sampling, spatial sampling, and simulated annealing (GSS) algorithm [29]. The genetic distances between markers were calculated in centiMorgans (cM) based on recombination values using the Kosambi function. QTL analysis was performed using R/qtl software package [30]. The LOD threshold for each data set was established based on the permutation test $(1,000$ permutations; $P<0.05)$. QTLs with LOD values above this threshold were considered significant. The phenotypic variance explained (PVE) by the two QTLs was estimated using the following formula: $1-10^{-}$ $2 \mathrm{~L} \circ \mathrm{D} / n$ [31], where $\mathrm{n}$ was the sample size.

\section{RNA-seq}

RNA-seq analyses were performed using shrimp from four families: the mapping family (LV-1) and three additional genetically-distinct families (LV-2, LV-3, and LV-4). Our previous analysis indicated that the 24-h median lethal concentration (LC50) of $\mathrm{NaNO}_{2}$ was $194.6 \mathrm{mg} / \mathrm{L}, 196.3 \mathrm{mg} / \mathrm{L}, 174.3 \mathrm{mg} / \mathrm{L}$, and $250.8 \mathrm{mg} / \mathrm{L}$ for families LV-1, LV-2, LV-3, and LV-4, respectively (Additional file 2: Table S2).

We randomly selected 200 shrimp from each family. Each family group was subjected to the acute nitrite stress test (700 mg/L nitrite), as described above with minor modifications. Briefly, in each family group, we collected the 20 most nitrite-sensitive shrimp and the 20 most nitrite-tolerant shrimp. Nitrite sensitive shrimp were collected as soon as soon as they began to exhibit symptoms of toxicity (i.e., swimming out of balance). The hepatopancreases were removed from all collected shrimp, and pooled to generate nitritesensitive and nitrite-tolerant pooled samples for each family. After removal and pooling, hepatopancreas tissues were immediately frozen in liquid nitrogen and stored in an ultra-low temperature freezer $\left(-80^{\circ} \mathrm{C}\right)$ until RNA extraction. 
Total RNA was extracted from each pooled sample using TRIzol reagent (Invitrogen, USA), following the manufacturer's instructions. Residual genomic DNA was removed with DNase I (Invitrogen, USA). RNA integrity was evaluated using the Agilent Bioanalyzer 2100 system (Agilent Technologies, USA), and RNA concentration was measured using a ND2000 spectrophotometer (NanoDrop, USA). Sequencing libraries were constructed using NEBNext UltraTM RNA Library Prep Kits for Illumina (NEB, USA), following the manufacturer's instructions. The libraries were sequenced on an Illumina HiSeq 2500 platform and pairedend reads were generated. Library construction and sequencing were performed at Biomarker Technologies Corporation (Beijing, China).

Raw sequencing reads were cleaned using in-house Perl scripts to remove reads containing adapter or poly$\mathrm{N}$ sequences, as well as low-quality (sequencing error rate $>0.1 \%$ ) reads. Clean reads were mapped to the $L$. vannamei genome using Hisat2 2.1.0 (http://ccb.jhu.edu/software/hisat2/index.shtml) [32]. Unique mapped reads with one or zero mismatches were used to calculate gene expression levels.

We used the DESeq2 package [33] to identify statistical differences in gene expression between samples. Genes were considered significantly differentially expressed when the false discovery rate (FDR) was $<0.01$ and fold change was $\geq 2$.

The DEGs were annotated against the following databases: $\mathrm{Nr}$ (ftp://ftp.ncbi.nih.gov/blast/db/), GO (http://www.geneontology.org/) and KEGG (http://www.genome.jp/kegg/).

\section{Candidate gene analysis}

The DEG sequences were compared to the $L$. vannamei genomic region within each QTL using the NCBI blast tool (https://blast.ncbi.nlm.nih.gov/Blast.cgi). We considered a DEG putatively associated with nitrite tolerance only if the DEG was located within the QTL interval, and the expression profile of the DEG was consistent across all four families (LV-1, LV-2, LV-3, and LV-4). Preliminary analysis identified a single gene meeting these criteria: SLC26A6.

\section{Verification of SLC26A6 expression using qRT-PCR}

To validate our RNA-seq results, we used qRT-PCR to quantify the expression of the candidate gene (SLC26A6) in the nitrite-tolerant and nitrite-sensitive pooled samples from the four $L$. vannamei families (LV-1, LV-2, LV-3, and LV-4). qRT-PCRs and RNA-seq analyses were performed using the same samples. qRTPCRs were performed with SYBR Premix Ex TaqTM II kits (TaKaRa, Japan), following the manufacturer's instructions. The primers used to detect SLC26A6 gene expression levels were RT-SLC-F and RT-SLC-R (Table 1). The qRT-PCR cycling program was as follows: preheating at $95^{\circ} \mathrm{C}$ for $30 \mathrm{~s}$, followed by 40 cycles of $95^{\circ} \mathrm{C}$ for $5 \mathrm{~s}$ and $60^{\circ} \mathrm{C}$ for $30 \mathrm{~s}$. We used L. vannamei $18 \mathrm{~S}$ RNA as the internal reference gene; this gene was amplified using the primers 18s-F and 18s-R [34] (Table 1). Three parallel qRT-PCRs were performed for each sample. Relative gene expression levels were calculated using the $2^{-\Delta \Delta C T}$ method [35].

\section{Effects of candidate gene silencing on nitrite tolerance}


The dsRNA used to silence the candidate gene (dsRNA-SLC26A6) was synthesized with the T7 RiboMAX Express RNAi System kit (Promega, USA), following the manufacturer's instructions, using the primers T7SLC26A6-F, T7-SLC26A6-R, SLC26A6-F and SLC26A6-R (Table 1). The primers T7-egfp-F, T7-egfp-R, egfp-F and egfp-R (Table 1) were used to synthesize dsRNA-egfp.

The dsRNA interference experiment was performed in 1000-liter glass saltwater tanks (30\%o salinity; $26-$ $27^{\circ} \mathrm{C} ; \mathrm{pH} 7.5-8.1$ ). We randomly selected $360 \mathrm{~L}$. vannamei from family LV-1. The selected shrimp were divided into three groups ( $n=40$ shrimp per group; three replicates of each group): buffer control, negative control, and experimental. Each shrimp in the buffer control group was injected at the second abdominal segment with $20 \mu \mathrm{g} 0.9 \%$ normal saline; each shrimp in the negative control group was injected with $20 \mu \mathrm{g}$ dsRNA-egfp; and each shrimp in the experimental group was injected with $20 \mu \mathrm{g}$ dsRNA-SLC26A6. Each shrimp was re-injected after $24 \mathrm{~h}$. At $12 \mathrm{~h}$ after the second injection, all shrimp were subjected to the acute nitrite stress test, as described above, for $120 \mathrm{~h}$. The time of death of each shrimp was recorded.

To evaluate the inhibitory effects of dsRNA on SLC26A6 gene expression, we randomly collected shrimp from the experimental group at $0 \mathrm{~h}, 36 \mathrm{~h}, 48 \mathrm{~h}$, and $72 \mathrm{~h}$ after the first injection of dsRNA-SLC26A6 (three shrimp were collected at each time point). The hepatopancreases were removed from the sampled shrimp as described above, and pooled by time point. Total RNA was extracted from each pool using a TRIzol Reagent kit (Invitrogen, USA), following the manufacturer's instructions. qRT-PCRs was performed using SYBR Premix Ex TaqTM II kits (TaKaRa, Japan), following the manufacturer's instructions. The primers used to detect SLC26A6 expression (SLC26A6-F and SLC26A6-R; Table 1) were designed using Primer Premier v 6.2 (http:/ / www.premierbiosoft) based on the SLC26A6 gene sequence (NCBI accession: XM_027371736.1) [27]. The qRT-PCR cycling program was as follows: preheating at $95^{\circ} \mathrm{C}$ for $30 \mathrm{~s}$, followed by 40 cycles of $95^{\circ} \mathrm{C}$ for $5 \mathrm{~s}$ and $60^{\circ} \mathrm{C}$ for $30 \mathrm{~s}$. We used L. vannamei $18 \mathrm{~S}$ RNA as the internal reference gene; this gene was amplified using the primers $18 \mathrm{~s}-\mathrm{F}$ and $18 \mathrm{~s}-\mathrm{R}$ (Table 1). Three biological replicates and three technical replicates were performed for each group. Relative mRNA expression levels were calculated using the $2^{-\triangle \triangle C T}$ method [35].

\section{Statistical analysis}

Data were shown as mean \pm standard deviation (SD), and analyzed using one-way ANOVAs in SPSS 13.0 [36]. We considered $P<0.01$ statistically significant.

\section{Results}

\section{Assessment of nitrite tolerance in the mapping family}

To measure nitrite tolerance, 157 shrimp from the L. vannamei mapping family (LV-1) were exposed to high concentrations of nitrite in seawater; survival time was recorded to indicate nitrite tolerance. All experimental shrimp died after 2-83 h of exposure. The average survival time was $43 \mathrm{~h}$. Individual survival time varied widely, and was normally distributed. Therefore, the nitrite-tolerance trait was suitable for QTL detection. 


\section{Construction of a high-density genetic linkage map}

We constructed L. vannamei SLAF libraries from the two parents and the 157 progeny tested for nitrate tolerance. High-throughput sequencing of the libraries yielded 262.12 gigabases $(\mathrm{Gb})$ of data, consisting of 1,310.33 million base (Mb) paired-end reads, each 100 bp in length (Additional file 3: Table S3). On average, the percentage of bases with a sequencing quality value $\geq 30$ (Q30) was $95.63 \%$, while the average GC content was $40.31 \%$. This indicated that the quality of the sequencing was good and the GC distribution was normal. In total, $59.34 \%$ of the paired-end reads mapped successfully to the $L$. vannamei genome. We used the rice (Oryza sativa japonica) genome as a control to estimate the validity of our library construction. For the rice library, we generated $343.21 \mathrm{Mb}$ of data (1.72 Mb paired-end reads), with a Q30 of $95.81 \%$ and a GC content of $40.96 \%$. In rice, $91.43 \%$ of the paired-end reads were mapped successfully to the rice genome. These results indicated that SLAF library construction and sequencing were normal.

After filtering and clustering, 1,079,516 SLAF markers were identified, with an average sequencing depth of 48.99-fold for the male parent, 46.61-fold for the female parent, and 13.19-fold for the offspring. After further filtering, we identified 219,463 polymorphic SLAF markers. These polymorphic markers were genotyped, and 101,907 were successfully encoded into eight genotypes (ab $\times \mathrm{cd}$, ef $\times$ eg, hk $\times \mathrm{hk}, \operatorname{Im} \times \mathrm{Il}$, $\mathrm{nn} \times \mathrm{np}$, aa $\times \mathrm{bb}, \mathrm{ab} \times \mathrm{cc}$, and $\mathrm{cc} \times \mathrm{ab}$ ). As the mapping population was an F1 population, polymorphic markers with the $a a \times b b$ segregation pattern (the homozygous genotype) were discarded.

After analyzing the linkages among the remaining SLAF markers, three maps (male, female, and sexaverage) were constructed. In total, 17,242 SLAF markers were labeled across all three genetic maps: 10,276 on the male map; 11,543 on the female map; and 17,242 on the sex-average map (Fig. 1). Each of the three maps contained 44 linkage groups (Additional file 4, 5 and 6: Tables S4, S5, and S6). The average genetic distance between markers on the male map was $0.60 \mathrm{cM}$ (total genetic distance: 6,164.79 cM); the average genetic distance between markers on the female map was $0.60 \mathrm{cM}$ (total genetic distance: $6,906.78 \mathrm{cM}$ ); and the average genetic distance between markers on the sex-average map was $0.4 \mathrm{cM}$ (total genetic distance: $6,828.06 \mathrm{cM}$ ).

\section{QTL mapping of nitrite tolerance}

Based on the genetic maps and the experimentally-collected nitrite-tolerance data for the mapping family, we performed a QTL analysis of the nitrite-tolerance trait. The threshold LOD score, above which QTLs were considered significant, was determined to be 3.0 based on 1,000 permutations $(P<0.05)$. Using this threshold, we identified two QTLs (QNT1 and QNT2) for nitrite tolerance (Fig. 2). The QTLs (LOD values: 3.00-3.35) were located on LG23 and LG44, with confidence intervals of 67.87-77.59 cM and 91.35-93.51 cM, respectively. The phenotypic variation explained by these QTLs was 8.42-10.31\% (Table 2).

\section{Transcriptomic profiles of nitrite-tolerant and nitrite-sensitive shrimp from different families}

To identify candidate genes associated with nitrite tolerance in L. vannamei, we generated eight cDNA libraries using mRNA extracted from the pooled hepatopancreases of the 20 most nitrite-tolerant and the most 20 nitrite-sensitive shrimp in four genetically-distinct families. After quality control, a total of $58.18 \mathrm{~Gb}$ 
of clean data were obtained. Using these data, we identified 2,002, 1,983, 1,954, and 1,867 differentially expressed genes (DEGs) between the nitrite-tolerant and nitrite-sensitive shrimp in the families LV-1, LV-2, LV-3, and LV-4, respectively (Additional file 7: Table S7). The DEGs were annotated based on the databases $\mathrm{Nr}$ (Non-redundant protein sequences), GO (Gene Ontology) and KEGG (http://www.genome.jp/kegg/). Notably, the GO and KEGG terms overrepresented in the DEGs were similar across all four families. The most overrepresented GO terms in the DEGs were "cell, cell part, binding, catalytic activity, metabolic process, cellular process, single-organism process" (Fig. 3), while the most overrepresented KEGG terms were "Protein processing in endoplasmic reticulum", "Phagosome" and "Longevity regulating pathway multiple species" (Fig. 4).

\section{Candidate gene analysis}

Based on our alignment of the DEGs with the QTL regions in LG23 and LG44, we found that 231 DEGs were located in a QTL interval (the expression profiles and annotations of these DEGs are listed in Additional file 8: Table S8). Of these DEGs, only one gene (LOC113819511) exhibited a consistent pattern of expression between the nitrite-tolerant and nitrite-sensitive groups across all four families. This gene was annotated as solute carrier family 26 member 6 (SLC26A6; [27]). SLC26A6 was located within the QTL interval of LG23, and was significantly upregulated in the most nitrite-tolerant shrimp compared to the most nitrite-sensitive shrimp across all the four families (Additional file 8: Table S8). The log2-fold changes in SLC26A6 gene expression level between the nitrite-sensitive and nitrite-tolerant shrimp were 1.14, 1.45, 1.06, and 1.89 in the LV-1, LV-2, LV-3, and LV-4 families, respectively (Fig. 5). Thus, SLC26A6 was considered a candidate gene associated with nitrite resistance.

\section{Verification of candidate gene expression using quantitative real-time PCR (qRT-PCR)}

The patterns of SLC26A6 gene expression in nitrite-tolerant and nitrite-sensitive pooled samples from the families LV-1, LV-2, LV-3, and LV-4 were similar to the patterns determined using RNA-seq: SLC26A6 gene expression was significantly greater in the nitrite-tolerant shrimp as compared to the nitrite-sensitive shrimp across all four families (Fig. 5). The log2-fold changes in SLC26A6 gene expression level between the nitrite-sensitive and nitrite-tolerant shrimp were 1.65, 1.99, 1.76, and 2.01 in the LV-1, LV-2, LV-3, and LV-4 families, respectively (Fig. 5).

\section{Effects of candidate gene silencing on nitrite tolerance in shrimp}

qRT-PCR analysis showed that in shrimp collected 36,48 , and $72 \mathrm{~h}$ after the injection of dsRNA, SLC26A6 mRNA expression was significantly $(p<0.01)$ inhibited in comparison to the buffer control group (injected with saline) and the negative control group (injected with dsRNA-egfp; Fig. 6). The median lethal time (LT50) of shrimp injected with dsRNA-SLC26A6 was $23.67 \pm 2.87 \mathrm{~h}$, which was significantly shorter than that of shrimp injected with dsRNA-egfp $(51.33 \pm 6.94 \mathrm{~h})$ and that of shrimp injected with normal saline $(55.67 \pm 4.03 \mathrm{~h})$ (Fig. 7). This indicated that the silencing of SLC26A6 gene expression significantly reduced nitrite tolerance in $L$. vannamei. 


\section{Discussion}

Although genetic maps for $L$. vannamei have been previously published, most of these were generated using AFLP or SSR markers alone or in combination with other markers [37]. In such genetic maps, the number of mapped markers is relatively small, and the average distance between adjacent markers is relatively long. For example, Zhang et al. constructed a genetic linkage map of L. vannamei using 816 AFLP and SSR markers; the average density of this map was $15.1 \mathrm{cM}$ [38]. Similarly, Andriantahina et al. constructed a genetic map of $L$. vannamei using 451 AFLP and SSR markers; the average density of this map was $7.6 \mathrm{cM}$ [39]. Here, we constructed a genetic linkage map of L. vannamei using 17,242 SLAF markers mapped to $44 \mathrm{LGs}$; the average density of this map was $0.40 \mathrm{cM}$. To our knowledge only one other study has used SLAF markers to create a genetic map of $L$. vannamei, but this previous study included only 6146 SNP markers, with an average distance of $0.7 \mathrm{cM}$ [40]. Therefore, our genetic map contained more markers and spanned longer genetic distances than previously available high-density genetic maps of $L$. vannamei. This may be because our sequencing depth was greater in this study.

We used these high-density genetic maps to investigate genes associated with nitrite tolerance in $L$. vannamei. Nitrite is one of the most common contaminants of aquaculture systems. Nitrite pollution has deleterious effects on shrimp, inhibiting growth and immunity, stimulating stress responses, and increasing disease risks; in some cases, high nitrite levels may even cause shrimp death [12, 13, 41-43]. As shrimp are an important aquaculture organism, it is therefore critical to identify the genetic mechanisms underlying nitrite tolerance in shrimp. To this end, we used our genetic map to identify two QTLs for nitrite tolerance in L. vannamei, located on LG23 and LG44 (LOD values: $3.00-3.71$; confidence intervals: $67.87-77.59 \mathrm{cM}$ and 91.35-93.51, respectively); these QTLs explained $8.42-10.31 \%$ of the phenotypic variation. To the best of our knowledge, this is the first identification of QTLs for nitrite tolerance in $L$. vannamei. The identification of these QTLs will support further studies aiming to develop new, nitrite-tolerant varieties of $L$. vannamei.

QTL analyses based on high-density genetic maps are commonly used to identify candidate genes associated with specific traits of interest [44]. However, QTL regions typically contain several hundred genes [45]. Comprehensive analysis of gene expression profiles can be effectively used in conjunction with QTLs to reduce the number of potential candidate genes. We thus performed RNA-seq analyses of four genetically-distinct $L$. vannamei families to identify candidate genes associated with nitrite-tolerance. We identified 7,806 genes that were differentially expressed between the nitrite-tolerant and nitrite-sensitive shrimp across all four families; of these, only a single gene, SLC26A6, was located in a QTL interval and consistently exhibited the same expression pattern irrespective of family. In RNA-seq and qRT-PCR analyses of all four families, SLC26A6 was upregulated in nitrite-tolerant shrimp, and downregulated in nitritesensitive shrimp. Consistent with these results, we found that, when SLC26A6 gene expression was silenced using dsRNA, shrimp LT50 during acute nitrite exposure decreased significantly as compared to unsilenced controls. This suggested that SLC26A6 expression was essential for nitrite tolerance in shrimp. SLC26A6 belongs to the ten-member SLC26 gene family; genes in this family encode anion exchangers and are able to transport a wide variety of monovalent and divalent anions [46]. SLC26A6 plays a key role in the transport of oxalate [47]. Mutant mice lacking SLC26A6 tend to develop calcium oxalate urolithiasis, because the secretion of intestinal oxalate in these mice is defective, resulting in increased net oxalate 
absorption [47]. Thus, intracellular anion secretion is decreased when SLC26A6 is downregulated, increasing net absorption. In shrimp, previous studies have shown that ammonia secretion is closely related to ammonia tolerance [3]. Ammonia-tolerant shrimp are more able to excrete and detoxify ammonia, while also having an accelerated energy metabolism to provide sufficient energy; these adaptive strategies may increase ammonia stress tolerance $[3,48]$. Thus, we hypothesize that the upregulation of SLC26A6 in nitrite-tolerant shrimp increases the capacity of these shrimp to excrete and tolerate nitrite; conversely, nitrite-sensitive shrimp, which express low levels of SLC26A6, are less able to excrete and tolerate high levels of nitrite. Thus, SLC26A6 may be a useful a candidate gene associated with nitrite tolerance.

\section{Conclusions}

We constructed high-density genetic maps of L. vannamei and identified QTLs associated with nitrite tolerance using the SLAF-seq method. By combining QTL and transcriptome analyses, we identified a candidate gene associated with nitrite tolerance. Our work increases our understanding of the molecular mechanisms underlying nitrite tolerance in shrimp, and provides a basis for molecular-marker-assisted shrimp breeding.

\section{Abbreviations}

QTL:quantitative trait locus; ammonia-N: ammonia nitrogen; cM: centimorgans; Gb: gigabases; Mb: megabases; SLAF-seq: specific length amplified fragment sequencing; SNPs: single nucleotide polymorphisms; LOD: logarithm of odds; PCRs: polymerase chain reactions; qRT-PCR: quantitative realtime PCR; FPKM: fragments per kilobase of transcript per million mapped reads; FDR: false discovery rate; DEGs: differentially expressed genes; LGs: linkage groups

\section{Declarations}

\section{Acknowledgments}

We thank the Guangxi Shrimp Breeding Engineering Technology Research Center for providing animal materials.

\section{Authors' contributions}

$\mathrm{YZ}$ and $\mathrm{XHC}$ conceived the study. DZ and XLC wrote the manuscript and contributed in the bioinformatics analysis. WZ, MP, and XC conducted the experiments and contributed for raw data analysis. QINGL, QIANGL, YL, HW, HL, and JL contributed in the bioinformatics analysis. All authors read and approved the final manuscript.

\section{Funding}

This work was supported by the scientific and technological innovation major project of guangxi (aa17204080-1), scientific and technological innovation major base of guangxi (aa17204088-1), guangxi 
scientific research and technology development project (ab16380189), national modern agriculture industry technology system project (nycytxgxcxtd-14-01), national modern agroindustry technology research system (cars-48), and natural science foundation of guangxi province (2018GXNSFAA294140).

\section{Availability of data and materials}

All data generated or analysed during this study are included in this published article and its supplementary information files. Raw SLAF sequencing reads are deposited in NCBI database under the accession numbers PRJNA545877. Raw RNA-seq are deposited in NCBI database under the accession numbers SRR9822088, SRR9822087, SRR9822086, SRR9822092, SRR9822089, SRR9822096, SRR9822110, and SRR9822097.

\section{Ethics approval and consent to participate}

Not applicable

\section{Consent for publication}

Not applicable

\section{Competing interests}

The authors declare they have no competing interests.

\section{References}

1. Lu X, Kong J, Luan S, Dai P, Meng X, Cao B, Luo K: Transcriptome Analysis of the Hepatopancreas in the Pacific White Shrimp (Litopenaeus vannamei) under Acute Ammonia Stress. PloS one. 2016; 11(10):e0164396.

2. Javahery S, Noori A, Hoseinifar SH: Growth performance, immune response, and digestive enzyme activity in Pacific white shrimp, Penaeus vannamei Boone, 1931, fed dietary microbial lysozyme. Fish \& shellfish immunology. 2019; 92:528-535.

3. Xiao J, Li QY, Tu JP, Chen XL, Chen XH, Liu QY, Liu H, Zhou XY, Zhao YZ, Wang HL: Stress response and tolerance mechanisms of ammonia exposure based on transcriptomics and metabolomics in Litopenaeus vannamei. Ecotoxicology and environmental safety. 2019; 180:491-500.

4. Sun CB, Wang G, Chan SF: Effects of artificial infection of Litopenaeus vannamei by Micrococcus lysodeikticus and WSSV on the activity of immunity related enzymes. Fish \& shellfish immunology. 2015; 46(2):778-786.

5. Valencia-Castaneda G, Frias-Espericueta MG, Vanegas-Perez RC, Chavez-Sanchez MC, Paez-Osuna F: Toxicity of ammonia, nitrite and nitrate to Litopenaeus vannamei juveniles in low-salinity water in single and ternary exposure experiments and their environmental implications. Environmental toxicology and pharmacology. 2019. 
6. da Silva L, Pires NMM, Dong T, Teien HC, Yang Y, Storebakken T, Salbu B: The Role of Temperature, Ammonia and Nitrite to bioluminescence of Aliivibrio fischeri: towards a new sensor for aquaculture. Conference proceedings : Annual International Conference of the IEEE Engineering in Medicine and Biology Society IEEE Engineering in Medicine and Biology Society Annual Conference. 2018; 2018:4209-4212.

7. Cheng SY, Chen JC: Study on the oxyhemocyanin, deoxyhemocyanin, oxygen affinity and acid-base balance of Marsupenaeus japonicus following exposure to combined elevated nitrite and nitrate. Aquat Toxicol. 2002; 61(3-4):181-193.

8. Kim JH, Kang YJ, Kim KI, Kim SK: Toxic effects of nitrogenous compounds (ammonia, nitrite, and nitrate) on acute toxicity and antioxidant responses of juvenile olive flounder, Paralichthys olivaceus. Environmental toxicology and pharmacology. 2019; 67:73-78.

9. Chen JC, Lee Y: Effects of nitrite exposure on acid-base balance, respiratory protein, and ion concentrations of giant freshwater prawn Macrobrachium rosenbergii at low pH. Archives of environmental contamination and toxicology. 1997; 33(3):290-297.

10. Cheng SY, Chen JC: Joint action of elevated ambient nitrite and nitrate on hemolymph nitrogenous compounds and nitrogen excretion of tiger shrimp Penaeus monodon. Comparative biochemistry and physiology Toxicology \& pharmacology : CBP. 2002; 131(3):303-314.

11. Wasielesky WJ, Poersch LH, Martins TG, Miranda-Filho KC: Chronic effects of nitrogenous compounds on survival and growth of juvenile pink shrimp. Brazilian journal of biology $=$ Revista brasleira de biologia. 2017; 77(3):558-565.

12. Tseng IT, Chen JC: The immune response of white shrimp Litopenaeus vannamei and its susceptibility to Vibrio alginolyticus under nitrite stress. Fish \& shellfish immunology. 2004; 17(4):325-333.

13. Valencia-Castaneda G, Frias-Espericueta MG, Vanegas-Perez RC, Perez-Ramirez JA, Chavez-Sanchez MC, Paez-Osuna F: Acute Toxicity of Ammonia, Nitrite and Nitrate to Shrimp Litopenaeus vannamei Postlarvae in Low-Salinity Water. Bulletin of environmental contamination and toxicology. 2018; 101(2):229-234.

14. Valencia-Castaneda G, Frias-Espericueta MG, Vanegas-Perez RC, Chavez-Sanchez MC, Paez-Osuna F: Toxicity of ammonia, nitrite and nitrate to Litopenaeus vannamei juveniles in low-salinity water in single and ternary exposure experiments and their environmental implications. Environmental toxicology and pharmacology. 2019; 70.

15. Paez-Osuna F: The environmental impact of shrimp aquaculture: causes, effects, and mitigating alternatives. Environmental management. 2001; 28(1):131-140.

16. Zhu WY, Huang L, Chen L, Yang JT, Wu JN, Qu ML, Yao DQ, Guo CL, Lian HL, He HL et al: A HighDensity Genetic Linkage Map for Cucumber (Cucumis sativus L.): Based on Specific Length Amplified Fragment (SLAF) Sequencing and QTL Analysis of Fruit Traits in Cucumber. Frontiers in plant science. 2016; 7:437.

17. Wang Y, Sun S, Liu B, Wang H, Deng J, Liao Y, Wang Q, Cheng F, Wang X, Wu J: A sequence-based genetic linkage map as a reference for Brassica rapa pseudochromosome assembly. BMC genomics. $2011 ; 12: 239$. 
18. Okuda Y, Ueda J, Obatake Y, Murakami S, Fukumasa Y, Matsumoto T: Construction of a genetic linkage map based on amplified fragment length polymorphism markers and development of sequencetagged site markers for marker-assisted selection of the sporeless trait in the oyster mushroom (Pleurotus eryngii). Applied and environmental microbiology. 2012; 78(5):1496-1504.

19. Jones DB, Jerry DR, Khatkar MS, Raadsma HW, Zenger KR: A high-density SNP genetic linkage map for the silver-lipped pearl oyster, Pinctada maxima: a valuable resource for gene localisation and markerassisted selection. BMC genomics. 2013; 14:810.

20. Duran Y, Fratini R, Garcia P, Perez de la Vega M: An intersubspecific genetic map of Lens. TAG Theoretical and applied genetics Theoretische und angewandte Genetik. 2004; 108(7):1265-1273.

21. Maughan PJ, Bonifacio A, Jellen EN, Stevens MR, Coleman CE, Ricks M, Mason SL, Jarvis DE, Gardunia BW, Fairbanks DJ: A genetic linkage map of quinoa ( Chenopodium quinoa) based on AFLP, RAPD, and SSR markers. TAG Theoretical and applied genetics Theoretische und angewandte Genetik. 2004; 109(6):1188-1195.

22. Kuiper MT: Building a high-density genetic map using the AFLP technology. Methods Mol Biol. 1998; 82:157-171.

23. Poland JA, Brown PJ, Sorrells ME, Jannink JL: Development of high-density genetic maps for barley and wheat using a novel two-enzyme genotyping-by-sequencing approach. PloS one. 2012; 7(2):e32253.

24. Peterson BK, Weber JN, Kay EH, Fisher HS, Hoekstra HE: Double digest RADseq: an inexpensive method for de novo SNP discovery and genotyping in model and non-model species. PloS one. 2012; 7(5):e37135.

25. Sun X, Liu D, Zhang X, Li W, Liu H, Hong W, Jiang C, Guan N, Ma C, Zeng H et al: SLAF-seq: an efficient method of large-scale de novo SNP discovery and genotyping using high-throughput sequencing. PloS one. 2013; 8(3):e58700.

26. Gilcreas FW: Future of standard methods for the examination of water and wastewater. Health laboratory science. 1967; 4(3):137-141.

27. Zhang X, Yuan J, Sun Y, Li S, Gao Y, Yu Y, Liu C, Wang Q, Lv X, Ma KY et al: Penaeid shrimp genome provides insights into benthic adaptation and frequent molting. Nature communications. 2019; 10(1):356.

28. Li H, Durbin R: Fast and accurate short read alignment with Burrows-Wheeler transform. Bioinformatics. 2009; 25(14):1754-1760.

29. Liu D, Ma C, Hong W, Huang L, Liu M, Liu H, Zeng H, Deng D, Xin H, Song J et al: Construction and analysis of high-density linkage map using high-throughput sequencing data. PloS one. 2014; 9(6):e98855.

30. Broman KW, Gatti DM, Simecek P, Furlotte NA, Prins P, Sen S, Yandell BS, Churchill GA: R/qt12: Software for Mapping Quantitative Trait Loci with High-Dimensional Data and Multiparent Populations. Genetics. 2019; 211(2):495-502. 
31. Gardiner SE, Norelli JL, de Silva N, Fazio G, Peil A, Malnoy M, Horner M, Bowatte D, Carlisle C, Wiedow C et al: Putative resistance gene markers associated with quantitative trait loci for fire blight resistance in Malus 'Robusta 5' accessions. BMC genetics. 2012; 13:25.

32. Kim D, Langmead B, Salzberg SL: HISAT: a fast spliced aligner with low memory requirements. Nature methods. 2015; 12(4):357-360.

33. Love MI, Huber W, Anders S: Moderated estimation of fold change and dispersion for RNA-seq data with DESeq2. Genome biology. 2014; 15(12):550.

34. Zhang Q, Li F, Zhang X, Dong B, Zhang J, Xie Y, Xiang J: cDNA cloning, characterization and expression analysis of the antioxidant enzyme gene, catalase, of Chinese shrimp Fenneropenaeus chinensis. Fish \& shellfish immunology. 2008; 24(5):584-591.

35. Livak KJ, Schmittgen TD: Analysis of relative gene expression data using real-time quantitative PCR and the 2(-Delta Delta C(T)) Method. Methods. 2001; 25(4):402-408.

36. Link J, Pachaly J: [Intranarcotic infusion therapy - a computer interpretation using the program package SPSS (Statistical Package for the Social Sciences)]. Infusionstherapie und klinische Ernahrung. 1975; 2(4):255-259.

37. Abdelrahman H, ElHady M, Alcivar-Warren A, Allen S, Al-Tobasei R, Bao L, Beck B, Blackburn H, Bosworth B, Buchanan J et al: Aquaculture genomics, genetics and breeding in the United States: current status, challenges, and priorities for future research. BMC genomics. 2017; 18(1):191.

38. Zhang L, Yang C, Zhang Y, Li L, Zhang X, Zhang Q, Xiang J: A genetic linkage map of Pacific white shrimp (Litopenaeus vannamei): sex-linked microsatellite markers and high recombination rates. Genetica. 2007; 131(1):37-49.

39. Andriantahina F, Liu X, Huang H: Genetic map construction and quantitative trait locus (QTL) detection of growth-related traits in Litopenaeus vannamei for selective breeding applications. PloS one. 2013; 8(9):e75206.

40. Yu Y, Zhang X, Yuan J, Li F, Chen X, Zhao Y, Huang L, Zheng H, Xiang J: Genome survey and highdensity genetic map construction provide genomic and genetic resources for the Pacific White Shrimp Litopenaeus vannamei. Scientific reports. 2015; 5:15612.

41. Duan Y, Zhang J, Wang Y, Liu Q, Xiong D: Nitrite stress disrupts the structural integrity and induces oxidative stress response in the intestines of Pacific white shrimp Litopenaeus vannamei. Journal of experimental zoology Part A, Ecological and integrative physiology. 2018; 329(1):43-50.

42. Guo H, Xian JA, Wang AL: Analysis of digital gene expression profiling in hemocytes of white shrimp Litopenaeus vannamei under nitrite stress. Fish \& shellfish immunology. 2016; 56:1-11.

43. Brown MN, Briones A, Diana J, Raskin L: Ammonia-oxidizing archaea and nitrite-oxidizing nitrospiras in the biofilter of a shrimp recirculating aquaculture system. FEMS microbiology ecology. 2013; 83(1):1725.

44. Cervino AC, Li G, Edwards S, Zhu J, Laurie C, Tokiwa G, Lum PY, Wang S, Castellani LW, Lusis AJ et al: Integrating QTL and high-density SNP analyses in mice to identify Insig2 as a susceptibility gene for plasma cholesterol levels. Genomics. 2005; 86(5):505-517. 
45. Wayne ML, McIntyre LM: Combining mapping and arraying: An approach to candidate gene identification. Proceedings of the National Academy of Sciences of the United States of America. 2002; 99(23):14903-14906.

46. Mount DB, Romero MF: The SLC26 gene family of multifunctional anion exchangers. Pflugers Archiv : European journal of physiology. 2004; 447(5):710-721.

47. Jiang Z, Asplin JR, Evan AP, Rajendran VM, Velazquez H, Nottoli TP, Binder HJ, Aronson PS: Calcium oxalate urolithiasis in mice lacking anion transporter Slc26a6. Nature genetics. 2006; 38(4):474-478.

48. Shan H, Geng Z, Ma S, Wang T: Comparative study of the key enzymes and biochemical substances involved in the energy metabolism of Pacific white shrimp, Litopenaeus vannamei, with different ammonia-N tolerances. Comparative biochemistry and physiology Toxicology \& pharmacology : CBP. 2019; 221:73-81.

\section{Tables}

Table 1. Primers used in this study.

\begin{tabular}{|c|c|}
\hline \multicolumn{2}{|c|}{ Name $\quad$ Sequence $\left(5^{\prime}-3^{\prime}\right)$} \\
\hline \multicolumn{2}{|c|}{ Primers for quantitative real-time PCR } \\
\hline $18 \mathrm{~s}-\mathrm{F}$ & СTCTGCTGAACCGCATTACTTG \\
\hline $18 s-R$ & TGCCGAGGGTTTTGGTCT \\
\hline \multicolumn{2}{|c|}{ RT-SLC26A6-F AGATAGCGTTCGGTCTGCTG } \\
\hline \multicolumn{2}{|c|}{ RT-SLC26A6-R GAGGGACTTGACCTGTGACG } \\
\hline \multicolumn{2}{|c|}{ Primers for gene silencing } \\
\hline SLC26A6- & GAGAAGCGAAGTTTGTGCG \\
\hline SLC26A6- & CGTCGTGAAGCCTGAGATG \\
\hline T7-SLC26 & F GGATCCTAATACGACTCACTATAGGGAGAAGCGAAGTTTGTGCG \\
\hline T7-SLC26 & R GGATCCTAATACGACTCACTATAGGCGTCGTGAAGCCTGAGATG \\
\hline T7-egfp-F & GGATCCTAATACGACTCACTATAGGGTGCCCATCCTGGTCGAGCT \\
\hline T7-egfp-R & GGATCCTAATACGACTCACTATAGGTGCACGCTGCCGTCCTCGAT \\
\hline egfp-F & GTGCCCATCCTGGTCGAGCT \\
\hline egfp-R & TGCACGCTGCCGTCCTCGAT \\
\hline
\end{tabular}

Table 2. Quantitative trait loci (QTLs) for nitrite tolerance detected in the mapping family (L' 1).

\begin{tabular}{|c|c|c|c|c|}
\hline$\overline{\text { QTL }}$ & $\begin{array}{l}\text { Linkage } \\
\text { group }\end{array}$ & $\begin{array}{l}\text { Interval of QTL } \\
(\mathrm{cM})\end{array}$ & $\begin{array}{l}\text { Logarithm of the odds } \\
\text { (LOD) }\end{array}$ & $\begin{array}{l}\text { Phenotypic variance explained (PVE) by } \\
\text { QTL }\end{array}$ \\
\hline QNT1 & 23 & $67.87-77.59$ & $3.00-3.35$ & $8.42 \%$ \\
\hline QNT2 & 44 & $91.35-93.51$ & $3.00-3.12$ & $10.31 \%$ \\
\hline
\end{tabular}

\section{Figures}




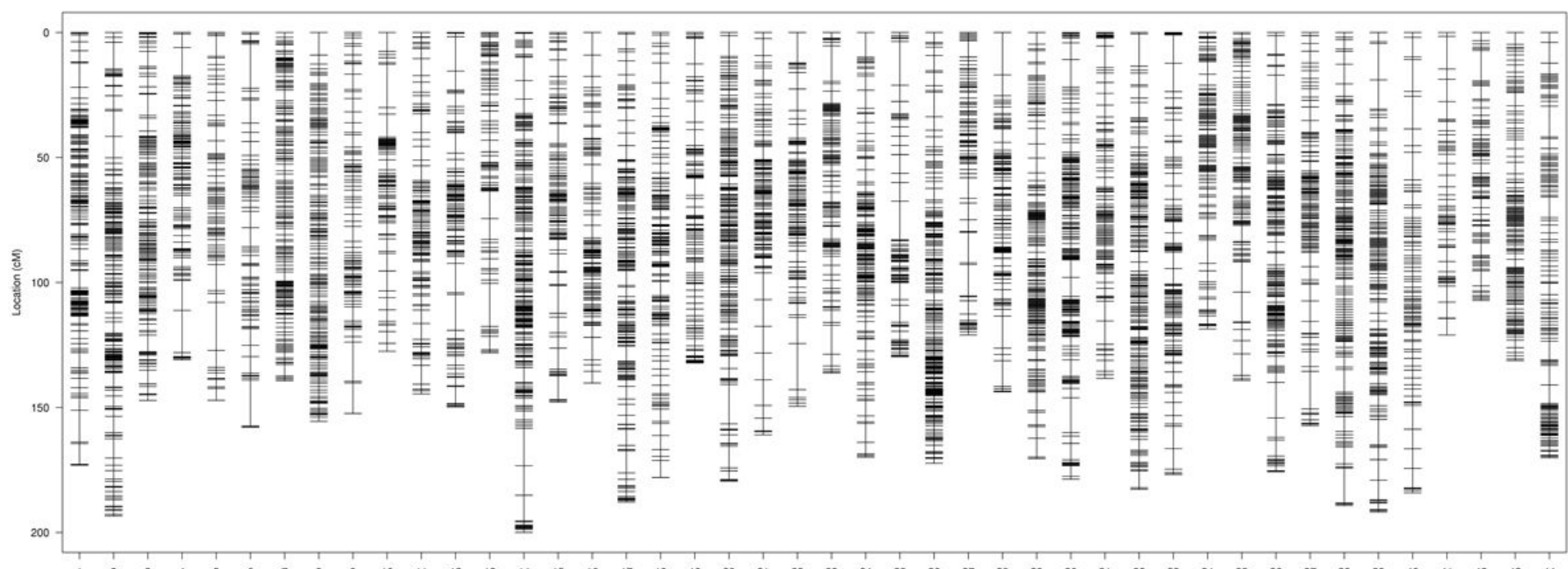

Linkage Group

Figure 1

High-density linkage sex-average map for Litopenaeus vannamei, showing genetic distances among specific length amplified fragment (SLAF) markers (shown as black bars).

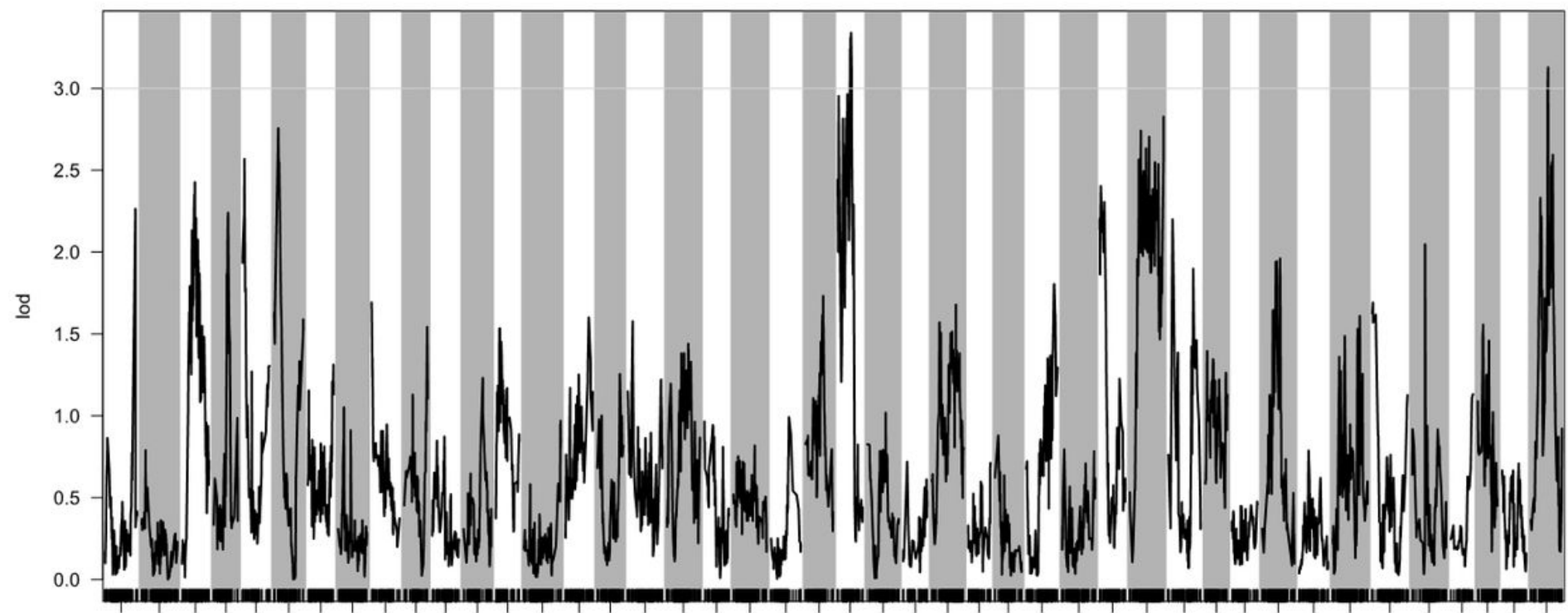

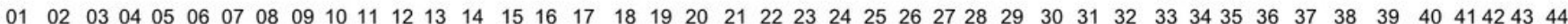
Chromosome

Figure 2

Quantitative trait loci (QTL) for nitrite tolerance in Litopenaeus vannamei, showing the logarithm of odds (LOD) values of the linkage groups. The gray line indicates the LOD threshold $(3.00 ; P=0.05)$. 


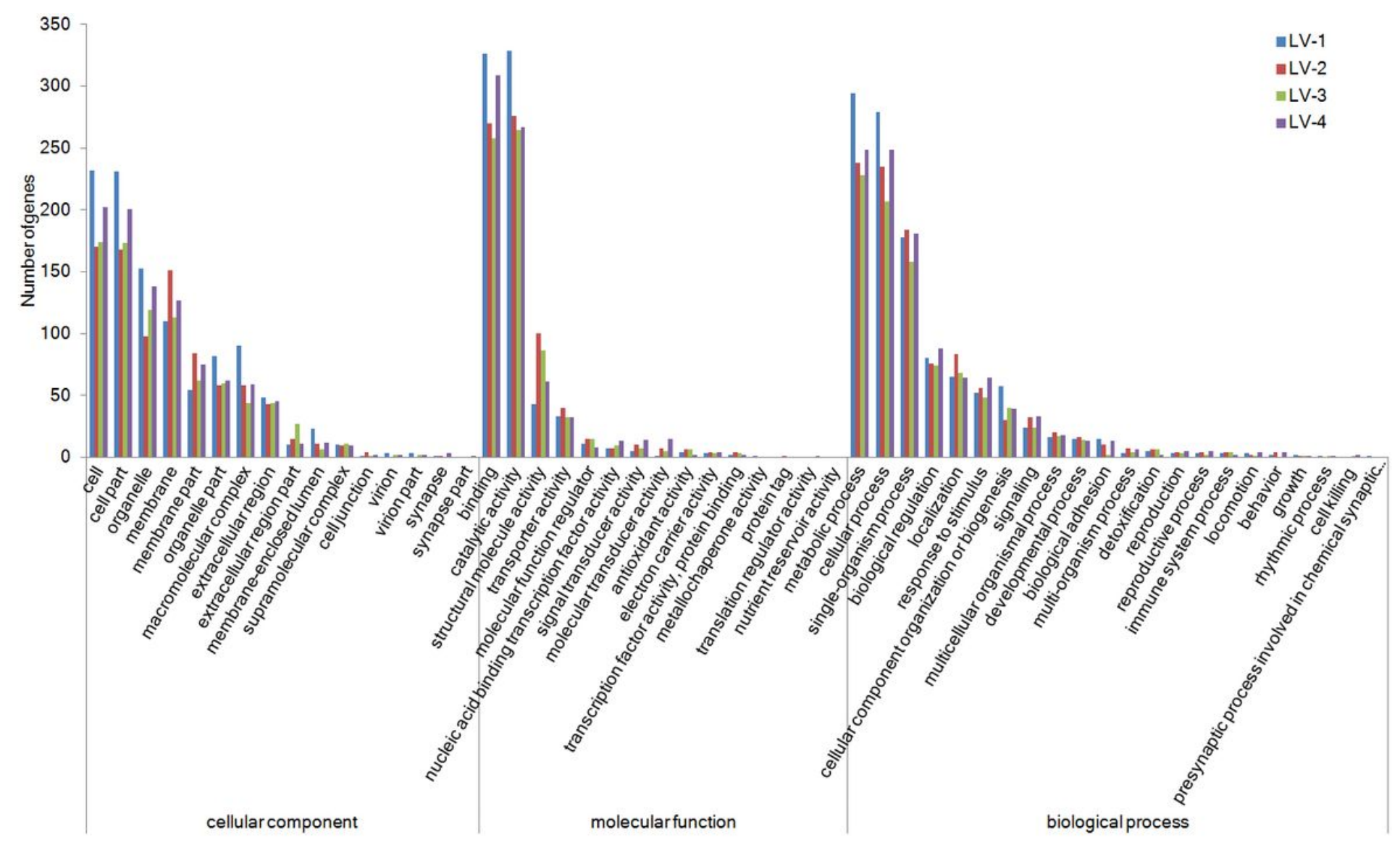

Figure 3

The Gene Ontology (GO) terms overrepresented in the differentially expressed genes (DEGs) across four genetically-distinct Litopenaeus vannamei families LV-1, LV-2, LV-3, and LV-4.

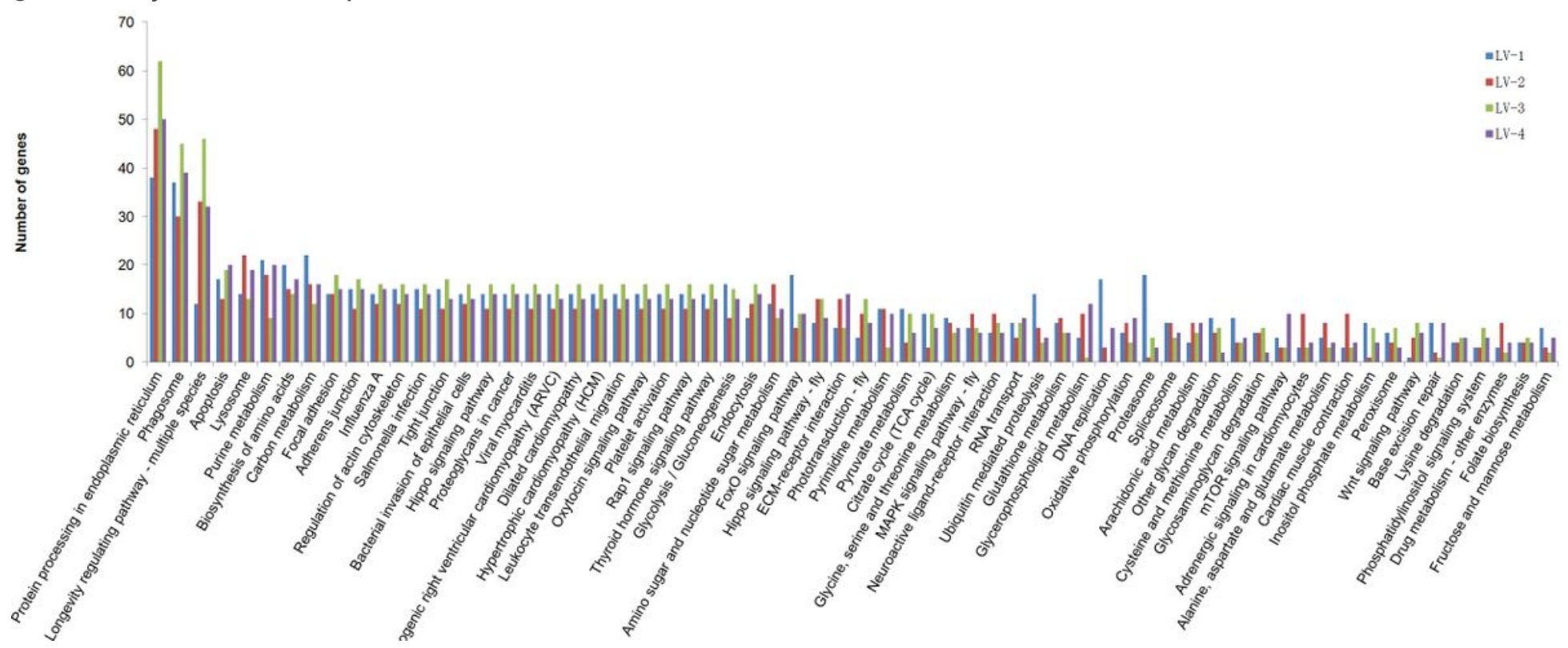

Figure 4 
The KEGG terms overrepresented in the in the DEGs across four genetically-distinct Litopenaeus vannamei families LV-1, LV-2, LV-3, and LV-4.

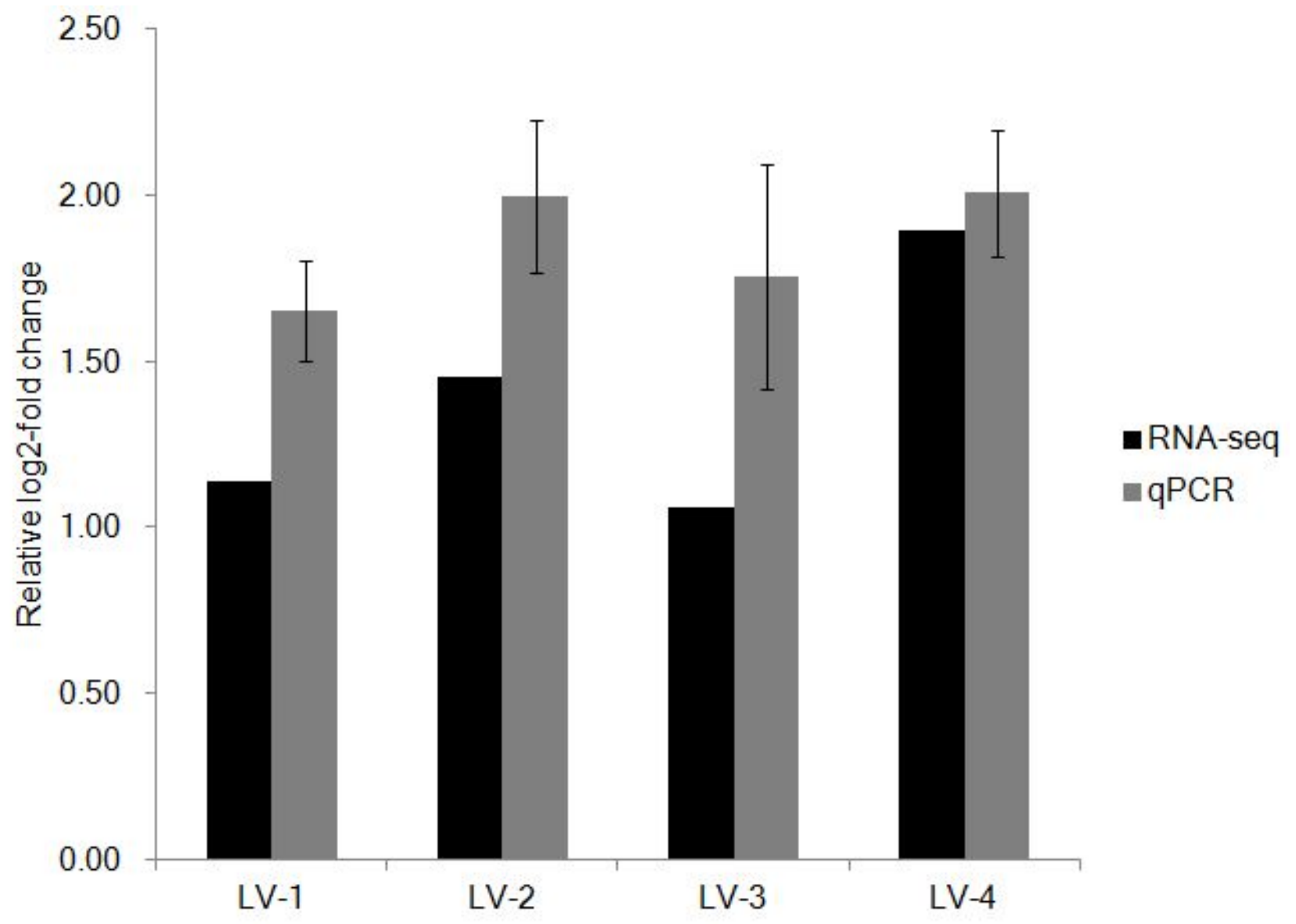

Figure 5

Expression of SLC26A6 from the transcriptomic analysis validated by qRT-PCR. Expression of SLC26A6 was detected in nitrite-sensitive and nitrite-tolerant shrimp from genetically-distinct Litopenaeus vannamei families LV-1, LV-2, LV-3, and LV-4. Data were normalized to 18s rRNA as the reference and presented as a relative log2-fold change to validate the transcriptomic analysis results. Error bars show the standard deviation of three technical replicates. 


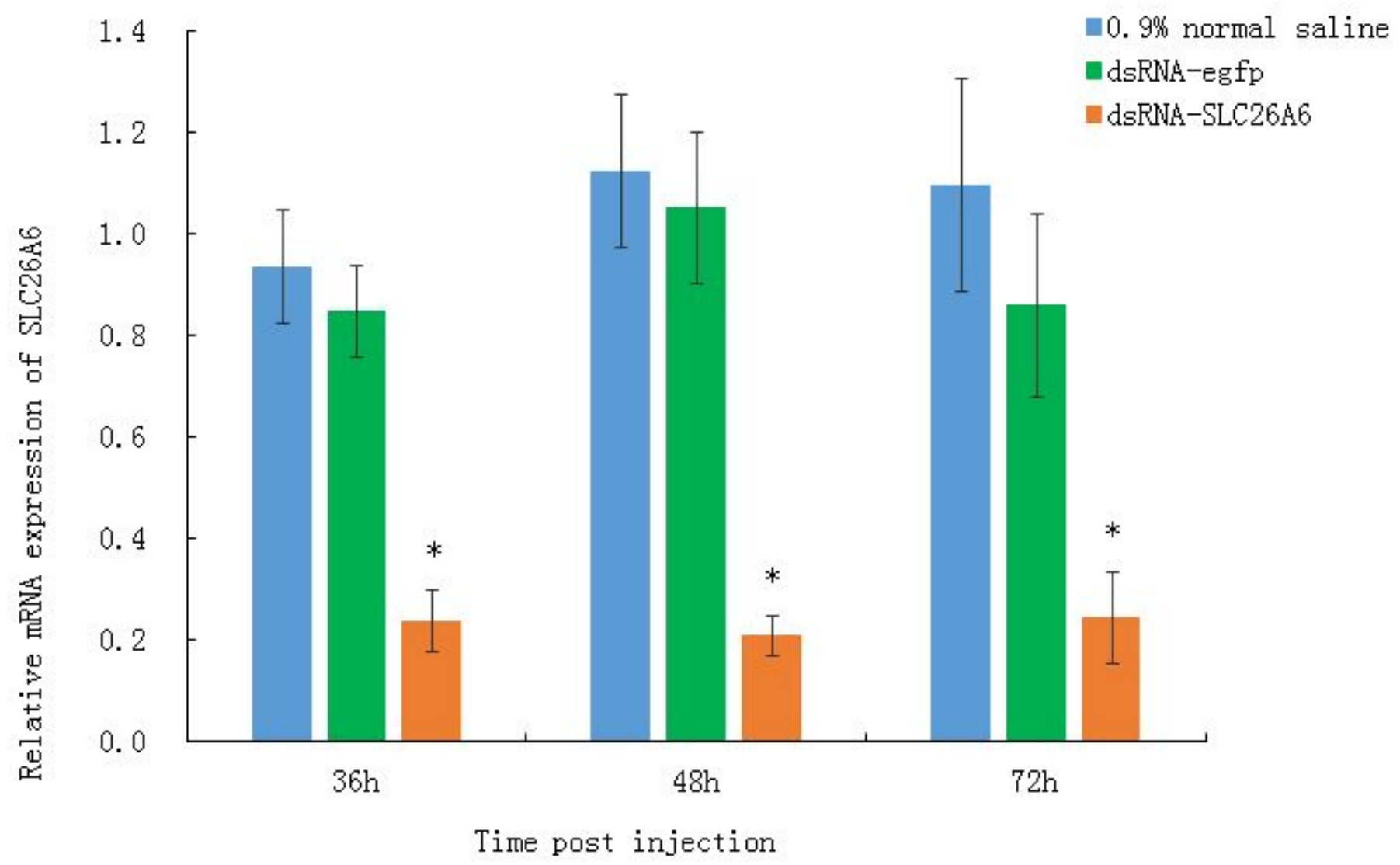

Figure 6

qRT-PCR quantification of relative SLC26A6 expression in shrimp from Litopenaeus vannamei family LV-1 at $36 \mathrm{~h}, 48 \mathrm{~h}$, and $72 \mathrm{~h}$ after injection with $0.9 \%$ normal saline (blue bars; buffer control), dsRNA-egfp (green bars; negative control), or dsRNA-SLC26A6 (orange bars; experimental group). L. vannamei 18S RNA was used as the internal reference gene. Asterisks $\left(^{*}\right)$ above bars indicate significant differences $(P<0.01)$ in relative gene expression as compared to both controls. Error bars show the standard deviation of three biological replicates. 


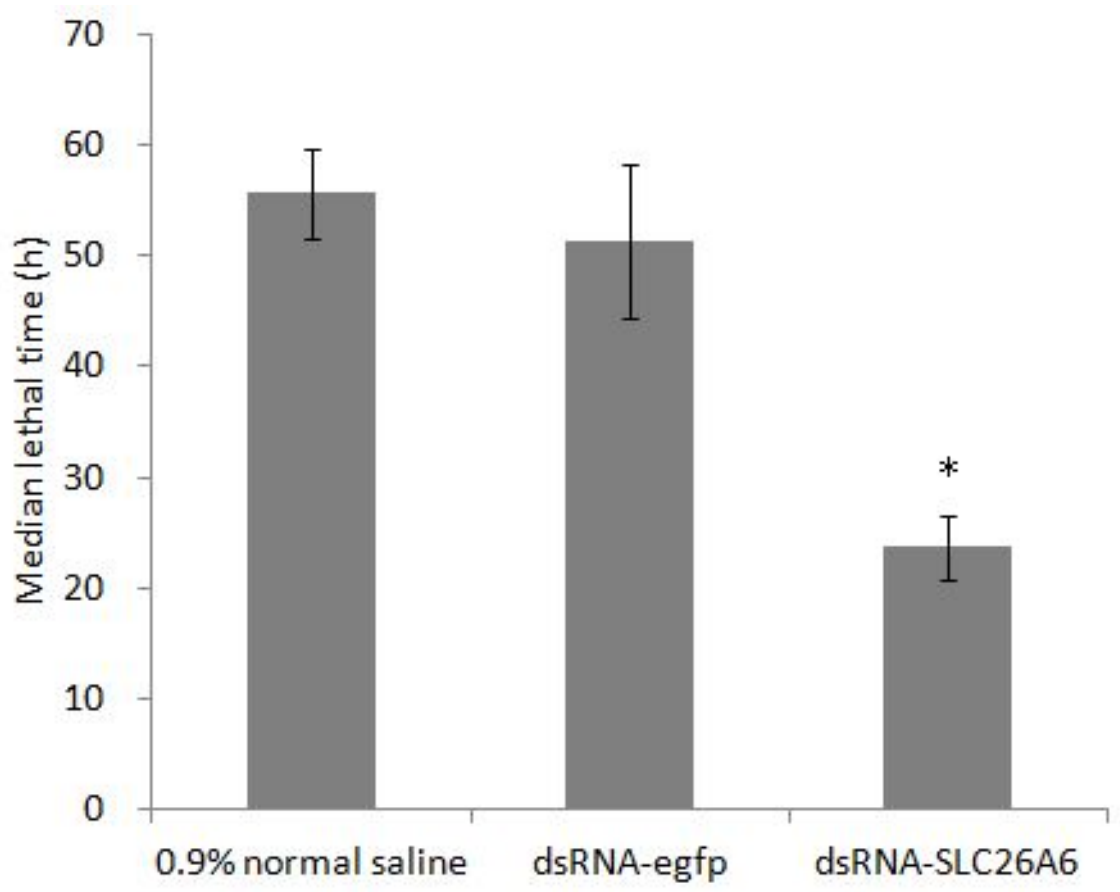

Figure 7

Median lethal time of Litopenaeus vannamei after injection with $0.9 \%$ normal saline (buffer control), dsRNA-egfp (negative control), or dsRNA-SLC26A6 (experimental group). Asterisks ( ${ }^{*}$ ) above bars indicate significant differences $(P<0.01)$ in relative gene expression as compared to both controls. Error bars show the standard deviation of three biological replicates.

\section{Supplementary Files}

This is a list of supplementary files associated with this preprint. Click to download.

- SupplementarytableS4.docx

- SupplementarytableS8.xlsx

- SupplementarytableS3.docx

- SupplementaryTableS7.docx

- SupplementarytableS5.docx

- SupplementarytableS6.docx

- SupplementarytableS1.docx

- SupplementaryTableS2.docx 